University of New Orleans

ScholarWorks@UNO

\title{
Enhanced spin-dependent tunneling magnetoresistance in magnetite films coated by polystyrene
}

\author{
Wendong Wang \\ University of New Orleans \\ Leszek Malkinski \\ University of New Orleans \\ Jinke Tang \\ University of New Orleans
}

Follow this and additional works at: https://scholarworks.uno.edu/phys_facpubs

Part of the Physics Commons

\section{Recommended Citation}

J. Appl. Phys. 101, 09F504 (2007)

This Article is brought to you for free and open access by the Department of Physics at ScholarWorks@UNO. It has been accepted for inclusion in Physics Faculty Publications by an authorized administrator of ScholarWorks@UNO.

For more information, please contact scholarworks@uno.edu. 


\title{
Enhanced spin-dependent tunneling magnetoresistance in magnetite films coated by polystyrene
}

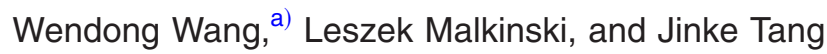 \\ Department of Physics, University of New Orleans, New Orleans, Louisiana 70148
}

\begin{abstract}
(Presented on 9 January 2007; received 31 October 2006; accepted 18 December 2006; published online 6 April 2007)

Hematite films were deposited by magnetron sputtering. A phase transformation from hematite to magnetite occurred when polystyrene (PS) coated hematite films were annealed above $200{ }^{\circ} \mathrm{C}$ in hydrogen flow. Giant negative magnetoresistance (MR) was observed with the best MR ratio of over $8 \%$ (at room temperature and in a field of $5.5 \mathrm{~T}$ ) found in samples annealed at $230{ }^{\circ} \mathrm{C}$. The temperature dependence of the resistivity is characteristic of intergranular tunneling. After the PS layer was removed and the films annealed again at $230{ }^{\circ} \mathrm{C}$ in hydrogen flow, the resistivity increased by about one order of magnitude and the MR ratio decreased to $4.3 \%$. These data show that PS coating layer can protect magnetite films from oxidation and enhance interganular spin-dependent tunneling magnetoresistance. (C) 2007 American Institute of Physics.
\end{abstract}

[DOI: $10.1063 / 1.2712031]$

\section{INTRODUCTION}

There has been increased interest in the half-metallic magnetite $\left(\mathrm{Fe}_{3} \mathrm{O}_{4}\right)$ as its highly spin polarized nature (supposedly $\sim 100 \%)$ and high Curie temperature $\left(T_{C} \sim 840 \mathrm{~K}\right)$ are desirable for spin electronic device applications. ${ }^{1,2}$ In principle, a high spin polarization should result in large tunneling magnetoresistance (TMR), since the latter arises from spin-dependent transport between the ferromagnetic (FM) electrodes of tunnel junctions, or FM grains of granular solids, and is proportional to the spin polarization of the tunneling electrons. ${ }^{3-5}$ Many studies have focused on the MR ratio in the magnetite of different forms including epitaxial and polycrystalline films, powders, and tunnel junctions. ${ }^{6-10} \mathrm{Un}$ fortunately, in most cases the MR ratio is much smaller than expected, especially at room temperature. In fact, it is well known that in polycrystalline specimens and powder compacts of magnetite, the surfaces or interfaces at the grain boundaries have rather different magnetic properties and reduced spin polarization than the bulk. ${ }^{11-14}$ This is a consequence of the off-stoichiometry, surface reconstruction, strain, defects, and bonding effects located at or close to the surfaces and interfaces. We have previously reported that polystyrene (PS) coated magnetite nanoparticles exhibit drastically enhanced intergranular tunneling magnetoresistance. ${ }^{15}$ The large TMR results from the better protection of the surfaces of $\mathrm{Fe}_{3} \mathrm{O}_{4}$ from oxidation by the PS coating. In this article we report our investigation of magnetite films, where PS coating was applied to the entire film rather than individual particles. The TMR is enhanced over uncoated films and reaches $8 \%$ at an applied field of $5.5 \mathrm{~T}$ at room temperature.

\section{RESULTS AND DISCUSSION}

Iron oxide films were deposited on glass substrates at room temperature by magnetron sputtering an iron target in a

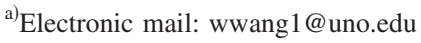

mixture of argon and oxygen gases. The base pressure of the chamber was lower than $2 \times 10^{-7}$ Torr, and the pressure during the deposition was about $1 \times 10^{-5}$ Torr. The thickness of the films was about $180 \mathrm{~nm}$. To coat the polymer layer, PS was first dissolved in xylene, then the solution was dropped on the films. After evaporating the solvent, a thin layer of PS was formed on the iron oxide films. The samples were then annealed in pure hydrogen flow.

$\mathrm{X}$-ray diffraction (XRD) spectra were collected with a Philips X'Pert diffractometer using $\mathrm{Cu} K \alpha$ radiation. Figure 1 shows the diffraction pattern of uncoated samples. The as-deposited samples are of hematite structure [Fig. 1(a)]. When they were annealed above $300{ }^{\circ} \mathrm{C}$ in pure hydrogen flow, a phase transformation occurred and the XRD pattern [Fig. 1(b)] indicates that it should be magnetite or $\gamma-\mathrm{Fe}_{2} \mathrm{O}_{3}$, although the two are often hard to distinguish from XRD. For PS coated iron oxide films, this transformation occurred when the annealing temperature was slightly higher than $200{ }^{\circ} \mathrm{C}$.

The resistance $(R)$ of the samples was measured with a standard four-point method. The as-deposited samples are insulators. After annealing in pure hydrogen, the films become conducting. Knowing that $\gamma-\mathrm{Fe}_{2} \mathrm{O}_{3}$ is an insulator, the films after reduction in hydrogen are magnetite.

Figure 2(a) shows the temperature dependence of the resistance for a PS coated sample annealed at $230{ }^{\circ} \mathrm{C}$. It exhibits an exponential increase with decreasing temperature. In Fig. 2(b), $R$ is plotted in a logarithmic scale as a function of $T^{-1 / 2}$. The linear behavior suggests that the transport mechanism is intergranular tunneling. ${ }^{5}$

The magnetoresistance of the samples was measured in a Quantum Design physical property measurement system up to a magnetic field of $5.5 \mathrm{~T}$. The magnetic field was applied perpendicular to the current direction. Negative giant magnetoresistance was observed and the MR ratio increases with increasing annealing temperature for the PS coated samples. The best MR ratio is over $8 \%$ at room temperature in an 


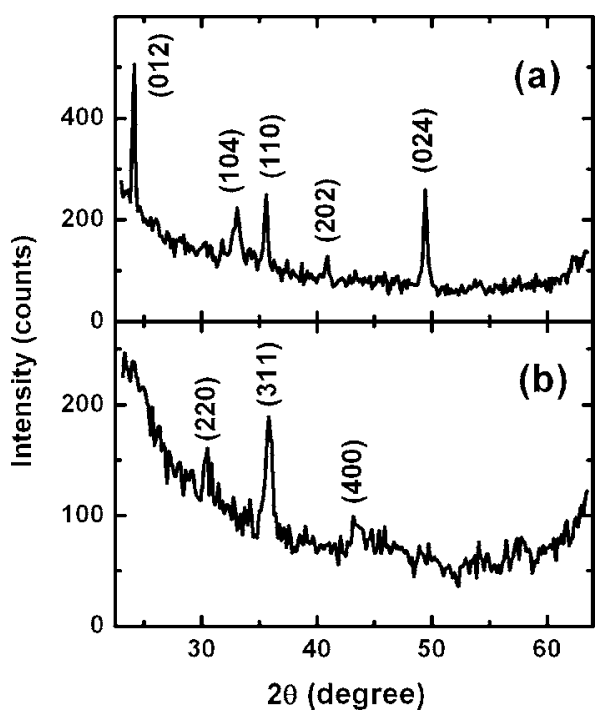

FIG. 1. X-ray diffraction pattern of iron oxide films (a) as-deposited and (b) annealed at $310{ }^{\circ} \mathrm{C}$ in hydrogen flow.

applied field of $5.5 \mathrm{~T}$ for the sample annealed at $230{ }^{\circ} \mathrm{C}$ [Fig. 3(a)]. However, after the PS layer was removed and the sample annealed again at $230{ }^{\circ} \mathrm{C}$ in hydrogen flow, the resistance of the sample increased by more than one order of magnitude and the MR ratio decreased down to $4.3 \%$, which is similar to most of the reported data on pressed magnetite powders and polycrystalline films. The MR ratio in such materials is typically about $4 \%-5 \%$ or lower at room temperature. ${ }^{9,16-18}$ This result indicates that although hydrogen of $99.999 \%$ purity was used in the annealing, the oxidation of the films at the grain boundaries was unavoidable, owing to the trace oxygen (about $2 \mathrm{ppm}$ ) when the PS cover was removed.

We believe that the oxidation should occur on the surface or at the grain boundaries of the film because it is well known that the surface state of magnetite is different and

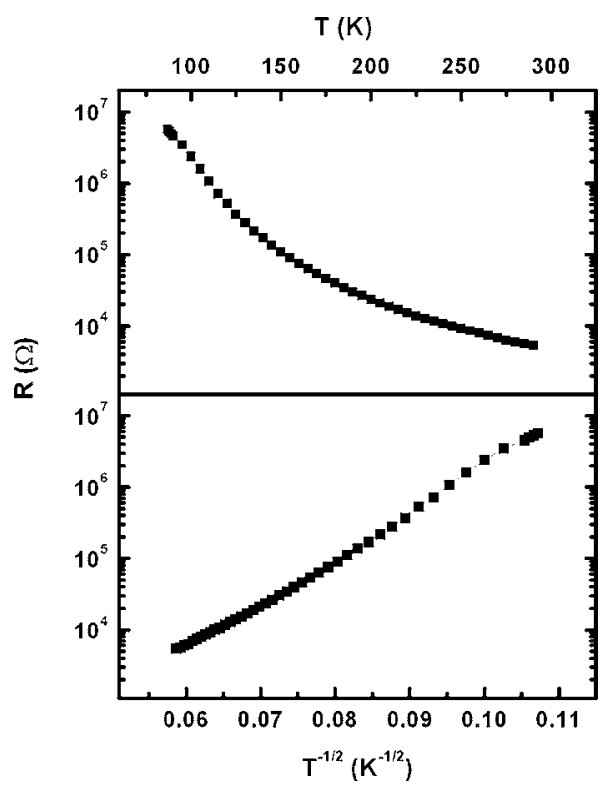

FIG. 2. Resistance as a function of temperature for PS coated sample annealed at $230{ }^{\circ} \mathrm{C}$ : (a) $\log R-T$ plot and (b) $\log R-T^{-1 / 2}$ plot.

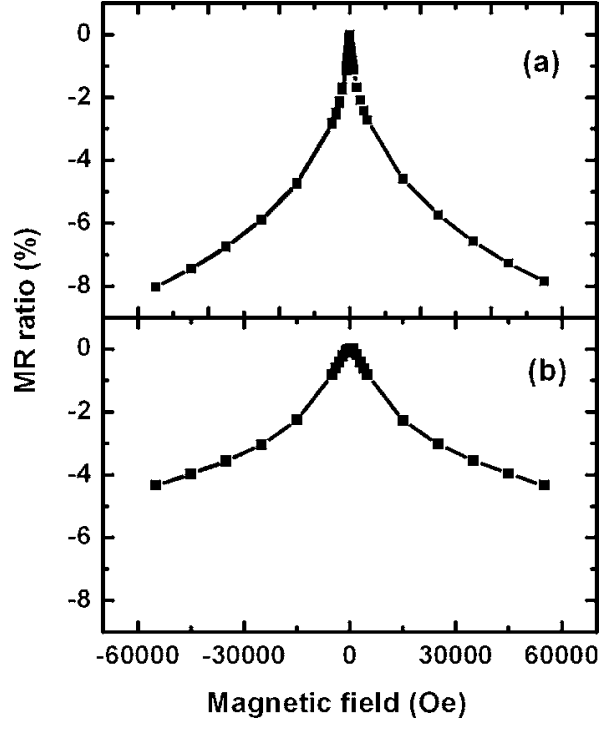

FIG. 3. Magneroresistance as a function of magnetic field for PS coated samples annealed at $230{ }^{\circ} \mathrm{C}$ : (a) with PS coating and (b) without PS coating.

more oxidized than the bulk. X-ray photoelectron spectroscopy (XPS) study of molecular beam epitaxy (MBE)-grown magnetite films indicates that the top two layers of surface are rich in Fe III compared to the bulk. ${ }^{19}$ Coating a PS layer on the iron oxide films can prevent the films from oxidation, thus limiting the degree to which the surface of $\mathrm{Fe}_{3} \mathrm{O}_{4}$ grains is altered away from the half-metallic state by oxidation. Therefore, the MR ratio of the samples with the PS coating layer is much higher than the samples without it. It also lowers the temperature at which the reduction takes place because PS should allow hydrogen to readily diffuse through but block most of oxygen. As mentioned earlier, the transformation temperature from hematite to magnetite decreases to $200{ }^{\circ} \mathrm{C}$ for PS coated films. Between 200 and $230{ }^{\circ} \mathrm{C}$, the increase of the annealing temperature favors the transformation to magnetite, resulting in the improvement of MR ratio.

Figure 4 shows the temperature dependence of MR ratio in an applied field of $5.5 \mathrm{~T}$ for the sample annealed at $230{ }^{\circ} \mathrm{C}$. In magnetite, there is a Verwey transition which is characterized by an increase in resistivity by about two orders of magnitude at the transition temperature $T_{V} \sim 120 \mathrm{~K}$. In our sample, MR ratio increases with the decrease of temperature above Verwey transition; however, it decreases below the transition. The drop of MR ratio below the Verwey

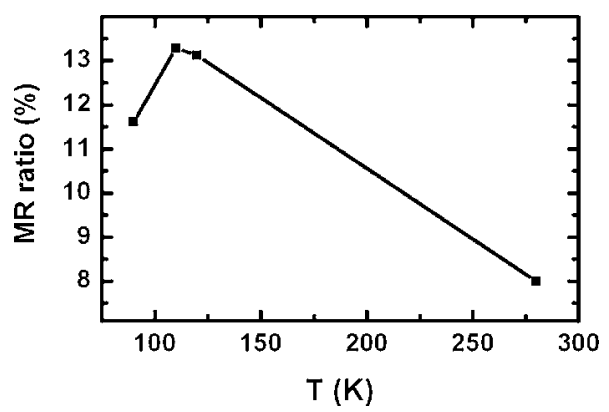

FIG. 4. Temperature dependence of MR ratio in an applied field of $5.5 \mathrm{~T}$ for PS coated sample annealed at $230^{\circ} \mathrm{C}$. 
point is probably attributed to the rapid increase in the resistivity of magnetite, which diminishes the spin-dependent tunneling current. ${ }^{15}$

For PS coated iron oxide films, the MR ratio sharply drops to about $0.5 \%$ after annealing at $240{ }^{\circ} \mathrm{C}$. The temperature dependence of the resistivity exhibits metallic behavior, which suggests that there may be precipitated metallic iron at this annealing temperature.

For the uncoated iron oxide films annealed at $310{ }^{\circ} \mathrm{C}$, the MR ratio is only about $2.1 \%$ at room temperature. We believe that this is because, although the annealing leads to the transformation from hematite to magnetite, the oxidation at grain boundaries of the films is unavoidable during cooling after the annealing, which reduces the MR ratio.

\section{CONCLUSIONS}

In summary, our experiments show that a PS layer can protect magnetite films from oxidation and enhance the spindependent intergranular tunneling magnetoresistance.

\section{ACKNOWLEDGMENT}

This work was supported by Sharp Laboratories of America and Louisiana Board of Regents Support Fund Grant No. LEQSF(2004-07)-RD-B-12.

${ }^{1}$ A. M. Bratkovsky, Phys. Rev. B 56, 2344 (1997).
${ }^{2}$ A. M. Bratkovsky, Appl. Phys. Lett. 72, 2334 (1998).

${ }^{3}$ S. Mitani, S. Takahashi, K. Takanashi, K. Yakushiji, S. Maekawa, and H. Fujimori, Phys. Rev. Lett. 81, 2799 (1998).

${ }^{4}$ X. W. Li, A. Gupta, G. Xiao, W. Qian, and V. P. Dravid, Appl. Phys. Lett. 73, 3282 (1998)

${ }^{5}$ J. Inoue and S. Maekawa, Phys. Rev. B 53, R11927 (1996).

${ }^{6}$ V. V. Gridin, G. R. Hearne, and J. M. Honig, Phys. Rev. B 53, 15518 (1996).

${ }^{7}$ W. Eerenstein, T. T. M. Palstra, S. S. Saxena, and T. Hibma, Phys. Rev. Lett. 88, 247204 (2002)

${ }^{8}$ W. Eerenstein, T. T. M. Palstra, T. Hibma, and S. Celotto, Phys. Rev. B 66, 20110(R) (2002).

${ }^{9}$ J. M. D. Coey, A. E. Berkowitz, L. Balcells, F. F. Putris, and F. T. Parker, Appl. Phys. Lett. 72, 734 (1998).

${ }^{10}$ D. L. Peng, T. Asai, N. Nozawa, T. Hihara, and K. Sumiyama, Appl. Phys. Lett. 81, 4598 (2002).

${ }^{11}$ J. M. De Teresa, A. Barhélémy, A. Fert, J. P. Contour, F. Montaigne, and P. Seneor, Science 286, 507 (1999).

${ }^{12}$ J. H. Park, E. Vescovo, H.-J. Kim, C. Kwon, R. Ramesh, and T. Venkatesan, Phys. Rev. Lett. 81, 1953 (1998).

${ }^{13}$ H. Dulli, E. W. Plummer, P. A. Dowben, J. Choi, and S. H. Liou, Appl. Phys. Lett. 77, 570 (2000).

${ }^{14}$ S. I. Rybchenko, Y. Fujishiro, H. Takagi, and M. Awano, Phys. Rev. B 72, 054424 (2005).

${ }^{15}$ W. Wang, M. Yu, M. Batzill, J. He, U. Diebold, and J. Tang, Phys. Rev. B 73, 134412 (2006).

${ }^{16}$ J. Tang, K.-Y. Wang, and W. Zhou, J. Appl. Phys. 89, 7690 (2001).

${ }^{17}$ H. Liu, E. Y. Jiang, H. L. Bai, R. K. Zheng, H. L. Wei, and X. X. Zhang, Appl. Phys. Lett. 83, 3531 (2003).

${ }^{18}$ D. Serrate, J. M. De Teresa, P. A. Algarabel, R. Fernández-Pacheco, J. Galibert, and M. R. Ibarra, J. Appl. Phys. 97, 084317 (2005).

${ }^{19}$ S. A. Chambers, S. Thevuthasan, and S. S. Joyce, Surf. Sci. 450, L273 (2000). 